\title{
Unfolding-induced in Haemoglobin by Exposure to Electromagnetic Fields: A FTIR Spectroscopy Study
}

\author{
EMANUELE CALABRÒ and SALVATORE MAGAZÙ \\ Department of Physics and of Earth Science, University of Messina, Italy. \\ ${ }^{*}$ Corresponding author E-mail: e.calabro@yahoo.com \\ http://dx.doi.org/10.13005/ojc/300104
}

(Received: January 01, 2014; Accepted: February 06, 2014)

\begin{abstract}
The effects of extremely low frequency electromagnetic field on the secondary structure of hemoglobin were investigated by means of Fourier Transform Infrared Spectroscopy. A decrease in intensity of the $\alpha$-helix component in the amide I and amide II regions was observed after exposure of $4 \mathrm{~h}$ to a $50 \mathrm{~Hz}$ electromagnetic field at $1 \mathrm{mT}$. In addition, Fourier self deconvolution analysis was carried out on exposed and not-exposed spectra. A relative increase of the $\beta$-sheet feature in the amide I region was evidenced, showing that an unfolding process of the protein occurred after exposure to extremely low frequency electromagnetic field, suggesting the hypothesis of the formation of aggregates.
\end{abstract}

Key words: Electromagnetic Field, FTIR Spectroscopy, Haemoglobin, Amide I region, unfolding.

\section{INTRODUCTION}

Several epidemiological studies have reported possible effects on human health from exposure to extremely low frequency electromagnetic field (ELF-EMF).

In response to public concern over health effects of EMF exposure, in 1996, the International EMF Project was established by WHO and the Radiation and Environmental Health Unit, which coordinated studies on EMF relative to the Environmental Health Criteria (EHC).
In particular, the International Agency for Research on Cancer (IARC) formally evaluated the evidence for carcinogenesis from exposure to static and ELF-EMFs, concluding that ELF-EMFs are possibly carcinogenic to humans ${ }^{1}$.

Milham and Ossiander have suggested that the appearance of the peak incidence at around age 3 in childhood acute lymphocytic leukemia is linked to electrification².

Further significant concern has been raised about the capacity of EMFs to cause DNA damage and chromosomes aberrations ${ }^{3-6}$. 
National radiation advisory authorities recommends measurements to minimize exposure to their citizens, as the exposure limits to electromagnetic fields recommended by the International Commission on Non-lonizing Radiation Protection (I.C.N.I.R.P.) $)^{7}$.

However, we focused our attention on proteins. Indeed, proteins are fundamentals in organic metabolism of livings. In the cells each protein must fold into the specific conformational state in a complex and highly crowded environment, and the folding process is aided by a range of auxiliary proteins ${ }^{8,9}$.

Otherwise it was largely demonstrated that several type of environmental stress agents can alter the secondary structure of proteins.

Recently it was proved that also ELFEMFs and MWs can alter the secondary structure of proteins ${ }^{10-16}$.

Haemoglobin is a heme-protein whose physiological importance is mainly related to its ability to bind molecular oxygen. The oxygen carried by heme-proteins is bound directly to the ferrous iron atom of the heme prosthetic group.

The heme portion of hemoglobin is extremely important because it aids in oxygen binding.

Haemoglobin is a tetrameric heme-protein found in erythrocytes where it is responsible for binding oxygen transporting the bound oxygen throughout the body to be used in aerobic metabolic functions.

The aim of this work was to investigate the alteration produced by exposure to EMFs on the secondary structure of this heme-protein that perform an important role in metabolism processes of organic systems, focusing the attention on aggregation mechanisms.

Fourier Transform Infrared (FTIR) Spectroscopy was used to investigate the effects of EMFs in the secondary structure of this protein in aqueous solution, in particular Amide I and II modes in the range $1700-1500 \mathrm{~cm}^{-1}$. Indeed, it may be considered the most versatile spectroscopic technique for analyzing the secondary structure of a protein in diverse physiochemical environments.

\section{MATERIALS AND METHODS}

\section{Haemoglobin samples were obtained as previously reported $^{10}$}

The exposure system for haemoglobin consisted of a couple of Helmholtz coils, with pole pieces of round parallel polar faces, to produce a uniform magnetic field at the center of the coils distance. A magnetic flux density of $1 \mathrm{mT}$ between the polar faces of the coils was generated by means of an AC voltage regulating up to $230 \mathrm{~V}$. Samples were placed at the center of a uniform field area between the coils and the magnetic field was continuously monitored by a magnetic field probe GM07 of HIRST Magnetic Instruments Ltd, UK.

a. Analogue unexposed samples at the same room temperature were used as the control.

b. FTIR spectra were recorded by a spectrometer, Vertex 80v, from Bruker Optics.

c. The attenuated total reflection (ATR) method was chosen for spectrum collection.

d. For each spectrum, 64 interferograms were collected with a spectral resolution of $4 \mathrm{~cm}^{-1}$ in the range from 4000 to $1200 \mathrm{~cm}^{-1}$, using the techniques accurately described in $^{11}$.

e. Either exposed or control samples were located in the same room at a temperature of $20^{\circ} \mathrm{C}$.

\section{RESULTS AND DISCUSSION}

\section{Exposure of Haemoglobin to $50 \mathrm{~Hz}$ EMF}

Samples of $250 \mu \mathrm{L}$ of hemoglobin in bidistilled water aqueous solutions were exposed for $4 \mathrm{~h}$ to a uniform electromagnetic field of $1 \mathrm{mT}$ at the frequency of $50 \mathrm{~Hz}$ at a room temperature of 20 ${ }^{\circ} \mathrm{C}$. Analogue unexposed samples at the same room temperature were used as the control.

Typical spectra from 1800 to $1400 \mathrm{~cm}^{-1}$, obtained after exposure, are showed in Figure 1.

The spectra exhibited an intense amide I band centered at about $1654 \mathrm{~cm}^{-1}$, corresponding mainly to an $\alpha$-helix structure content due to $\mathrm{C}=\mathrm{O}$ 
stretching vibration and a $\mathrm{N}-\mathrm{H}$ bending mode, a low intensity amide II, coupling of the $\mathrm{N}-\mathrm{H}$ bending and C-N stretching modes.

A significant decrease in intensity of the amide I and II modes was evidenced for exposed sample spectra, that can be due to a decrease of the $\alpha$-helix component, a loss of $\alpha$-helical and shortsegment connecting $\alpha$-helix segments in the amide I region.
In addition, a perceptible increase of the $\beta$-sheet content in the region $1635-1610 \mathrm{~cm}^{-1}$ can be observed in Figure 1, as well.

Hence, Fourier self deconvolution (FSD) analysis was used to highlight the alterations in the amide I region. The concept of FSD is based on the assumption which a spectrum of single narrow bands is broadened in the liquid or solid state and cannot be

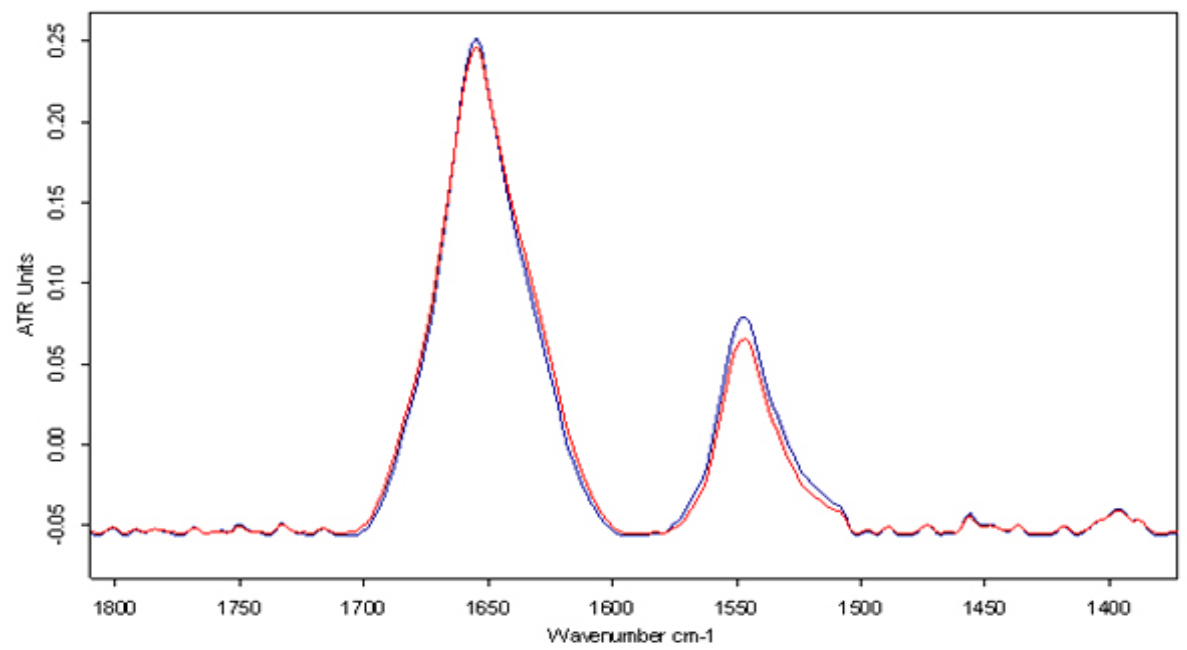

Fig. 1: Representative infrared spectra from 1800 to $1400 \mathrm{~cm}^{-1}$ of hemoglobin in bidistilled water solution after $4 \mathrm{~h}$ of exposure to $50 \mathrm{~Hz}$ frequency EMF at $1 \mathrm{mT}$ (red lines represent exposed samples spectra). The amide I and II regions are evidenced. The decrease in intensity after exposure was significant in particular in the amide II region

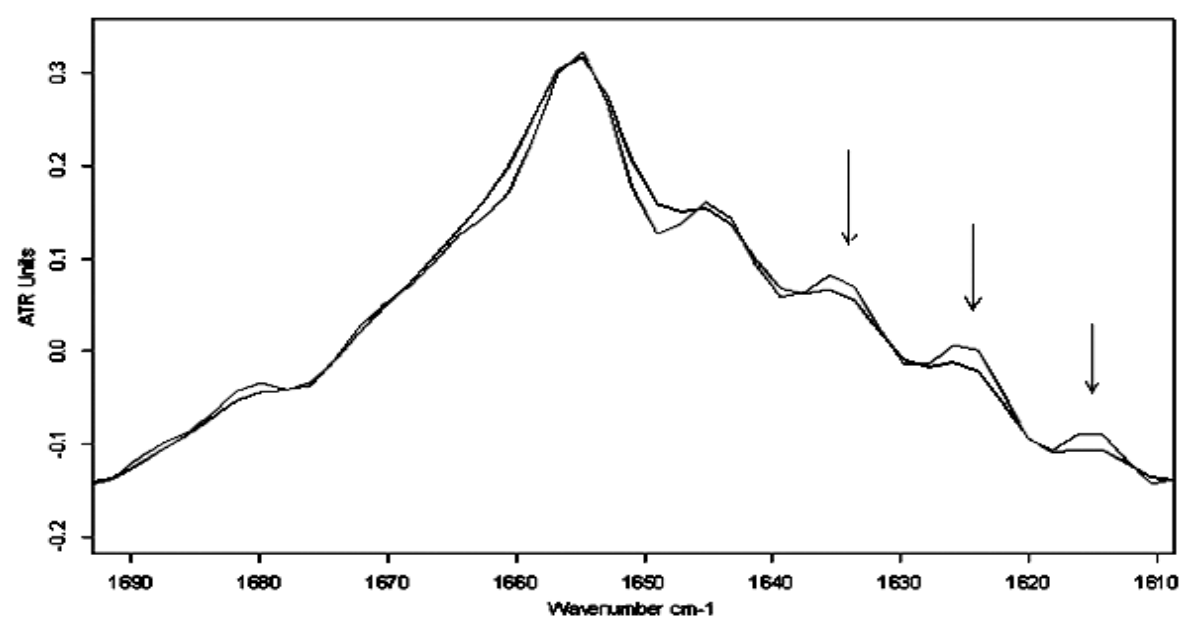

Fig. 2: Representative FSD infrared spectra in the amide I region of hemoglobin in bidistilled water solution after $4 \mathrm{~h}$ of exposure to $50 \mathrm{~Hz}$ frequency EMF at $1 \mathrm{mT}$.

The relative increases in intensity of $\beta$-sheet features (indicated by arrows) after exposure were evidenced by FSD analysis. Red lines represent exposed samples spectra 
distinguished in the amide envelope. A curve fitting procedure can be applied to estimate quantitatively the area of each component representing a type of secondary structure.

After FSD analysis on exposed and notexposed spectra, vector normalization was used.

This analysis revealed the presence of five vibration bands centered at 1654, and around 1635, 1625,1615 and $1685 \mathrm{~cm}^{-1}$ in the amide I region, as can be observed in Figure 2.

The band at $1654 \mathrm{~cm}^{-1}$ is due to $\alpha$-helix structures, and the other vibrations can be associated with $\beta$-sheet structures ${ }^{17-19}$.

This analysis relative to hemoglobin in bidistilled water aqueous solution revealed a significant increase in $\beta$-sheet bands after $4 \mathrm{~h}$ of exposure, indicated with arrows in Figure 2, comparing exposed and unexposed spectra. These features can be attributed to the formation of aggregates ${ }^{20-22}$.

In conclusion, an unfolding process of hemoglobin due to ELF-EMF exposure was enhanced and confirmed by FSD analysis.

\section{CONCLUSION}

The effects of exposure of $4 \mathrm{~h}$ to $50 \mathrm{~Hz}$ EMF at $1 \mathrm{mT}$ on hemoglobin aqueous solutions were studied by means of FTIR techniques, showing that ELF-EMF can affect infrared vibration bands of hemoglobin.

In particular, a loss of $\alpha$-helical and shortsegment connecting $\alpha$-helix segments was observed in amide I and amide II regions.

In addition, the use of FSD analysis evidenced a relative increase in intensity of the $\beta$-sheet content with respect to $\alpha$-helix component in the amide I region, that can be attributed to the formation of aggregates.

Previous literature have indicated that the propensity to cause the transition from $\alpha$-helix to $\beta$-sheet structure can be responsible for aggregation leading to the neurotoxicity and neurodegenerative disorders that can be considered as the first step to some pathologies. Hence, further research is needed to highlight bioprotective mechanisms against the effects of ELF-EMF.

\section{REFERENCES}

1. IARC Working Group on the Evaluation of Carcinogenic Risks to Humans. Non-ionizing radiation, Part 1: Static and extremely lowfrequency (ELF) electric and magnetic fields; Monographs on the Evaluation of Carcinogenic Risks to Humans, 80; IARC: Lyon (2002).

2. S. Milham, E.M. Ossiander, "Historical evidence that residential electrification caused the emergence of the childhood leukemia peak", Med. Hypotheses 56(3): 290-295 (2001).

3. I. Y. Belyaev, C. B. Koch, O. Terenius, K. Roxstrom- Lindquist, L. O. Malmgren, W. H. Sommer, L. G. Salford and B. R. Persson, "Exposure of Rat Brain to $915 \mathrm{MHz}$ GSM Microwaves Induces Changes in Gene
Expression but not Double Stranded DNA Breaks or Effects on Chromatin Conformation," Bioelectromagnetics, 27(4): 295-306 (2006).

4. E. Calabrò, S. Condello, S. Magazù and R. lentile "Electromagnetic fields low levels altered the DNA infrared region in RAdifferentiated SH-SY5Y neuroblastoma cells", BioTechnology: An Indian Journal 6(8-9): 267271 (2012).

5. E. Diem, C. Schwarz, F. Adlkofer, O. Jahn and $\mathrm{H}$. Rudiger, "Non-thermal DNA Breakage by Mobile-Phone Radiation $(1800 \mathrm{MHz})$ in Human Fibroblasts and in Transformed GFSH-R17 Rat Granulosa Cells in Vitro", Mutation Research 583(2): 178-183 (2005).

6. R. R. Tice, G. G. Hook, M. Donner, D. I. McRee and A.W. Guy, "Genotoxicity of Radiofrequency 
Signals. I. Investigation of DNA Damage and Micronuclei Induction in Cultured Human Blood Cells," Bioelectromagnetics 23(2): 113126 (2002).

7. International Commission on Non-lonizing Radiation Protection, "Guidelines for Limiting Exposure to Time -Varying Electric, Magnetic, and Electromagnetic Fields (up to $300 \mathrm{GHz}$ )," Health Physics, 74(7): 494-522, 1998.

8. R. J. Ellis and F. U. Hartl, "Principles of Protein Folding in the Cellular Environment," Current Opinion in Structural Biology, 9(1): 102-110 (1999).

9. M. J. Gething and J. Sambrook, "Protein Folding in the Cell," Nature, 355(6355): 33-45, 1992.

10. S. Magazù, E. Calabrò and S. Campo, "FTIR Spectroscopy Studies on the Bioprotective Effectiveness of Trehalose on Human Hemoglobin Aqueous Solutions under 50 $\mathrm{Hz}$ Electromagnetic Field Exposure," The Journal of Physical Chemistry B, 114(37): 12144-12149 (2010).

11. S. Magazù and $\mathrm{E}$. Calabrò, "Studying the Electromagnetic-Induced Changes of the Secondary Structure of Bovine Serum Albumin and the Bioprotective Effectiveness of Trehalose by FTIR Spectroscopy," The Journal of Physical Chemistry B, 115(21): 6818-6826 (2011).

12. E. Calabrò, S. Magazù, "Inspections of Mobile Phone Microwaves Effects on Proteins Secondary Structure by means of Fourier Transform Infrared Spectroscopy", Journal of Electromagnetic Analysis \& Applications 2(11): 607-617 (2010).

13. S. Magazù, E. Calabrò, S. Campo, S. Interdonato, "New Insights into Bioprotective Effectiveness of Disaccharides: a FTIR Study of Human Haemoglobin Aqueous Solutions exposed to Static Magnetic Fields", Journal of Biological Physics 38(1): 61-74 (2012). DOI: 10.1007/s10867-010-9209-1, published on-line March 9, 2011.

14. E. Calabrò and S. Magazù, "Electromagnetic Fields Effects on the Secondary Structure of Lysozyme and Bioprotective Effectiveness of Trehalose", Advances in Physical Chemistry, Article ID 970369, 6 pages, doi:10.1155/2012/970369, 2012 (2012).
15. E. Calabrò, S. Magazù, "Comparison between conventional convective heating and microwave heating: an FTIR spectroscopy study of the effects of microwave oven cooking of bovine breast meat", Journal of Electromagnetic Analysis \& Applications 4(11): 433-439 (2012).

16. E. Calabrò, S. Magazù, Unfolding and Aggregation of Myoglobin can be Induced by Three Hours Exposure to Mobile Phone Microwaves: a FTIR spectroscopy study, Spectroscopy Letters: An International Journal for Rapid Communication, 46(8): 583-589 (2013).

17. E. Calabrò, S.Magazù, Non-Thermal Effects of Microwave Oven Heating on Ground Beef Meat Studied in the Mid-Infrared Region by FTIR Spectroscopy, Spectroscopy Letters: An International Journal for Rapid Communication, published online 01 Aug 2013 DOI:10.1080/00387010.2013.828313 (2013).

18. Krishan, B. and Zaafarany, I., Orient J. Chem., 29(4): 1571-1577 (2013).

19. E. Calabrò, S. Condello, M. Currò, N. Ferlazzo, D. Caccamo, S. Magazù and R. lentile, Effects of Low Intensity Static Magnetic Field on FTIR spectra and ROS production in SH-SY5Y neuronal-like cells, Bioelectromagnetics 34 : 618-629 (2013).

20. E. Calabrò, S. Magazù, S. Campo, "Microwaveinduced increase of amide I and amide II vibration bands and modulating functions of sodium-chloride, sucrose and trehalose aqueous solutions: The case study of Haemoglobin", Research Journal of Chemistry and Environment, 16(4): 59-67 (2012).

21. A. A. Ismail, H. H. Mantch, P.T.T. Wong, "Aggregation of chymotrypsinogen: portrait by infrared spectroscopy", Biochim. Biophys. Acta 1121: 183-188 (1992).

22. T. Lefevre, M. Subirade, "Molecular differences in the formation and structure of finestranded and particulate $\beta$-lactoglobulin gels", Biopolymers 54: 578-586 (2000).

23. R. Bauer, R. Carrotta, C. Rischel, L. Ogendal, "Characterization and isolation of intermediates in $\beta$-lactoglobulin heat aggregation at high $\mathrm{pH}$ ", Biophys. J. 79: 1030-1038 (2000). 